\title{
Electronic Structure Analysis of a New Quinoid Conjugated Polymer
}

\author{
Ulrike Salzner
}

Department of Chemistry, Bilkent University, 06533 Bilkent, Ankara, Turkey. Tel.: (312) 290-2122; Fax: (312) $266-4579$. E-mail: salzner@fen.bilkent.edu.tr

Received: 19 October 1999/ Accepted: 14 January 2000/ Published: 28 February 2000

\begin{abstract}
The low lying unoccupied orbitals of oligomers of 4-dicyanomethylene-4H-cyclopenta[2,1b:3,4-b'] dithiophene (CDM) are not delocalized over the whole molecule. Is such electron localization in the conduction band of poly-CDM responsible for its low n-type conductivity? Are polymers of the tricyclic thioketone (TCT) with more delocalized unoccupied orbitals a better alternative for stable ndopable conducting polymers?

Monomer through tetramer of TCT have been optimized with density functional theory. IP, EA, energy gap, and band width of the corresponding polymer were obtained by extrapolation. Comparison with data for oligomers of 4-dicyanomethylene-4H-cyclopenta[2,1-b:3,4-b'] dithiophene and of thiophene indicates that the novel polymer would have a small band gap and would fulfil the conditions for ndopability and high mobility of n-type carriers.
\end{abstract}

Keywords Low band gap polymers, Band widths, Thioketones, Conductivity, Density functional theory

\section{Introduction}

Although research on conducting polymers[1] started with the discovery of intrinsically conducting polysulfurnitride,[2] no other polymer with intrinsic metallic conductivity has been discovered since. Currently, conducting polymers like polyaniline, polythiophene, polypyrrole, and polyparaphenylene are among the most studied because of their chemical stability. All of these systems are semiconductors that require doping to become conducting. P-doping works well and conductivities of up to $2000 \mathrm{~S} \mathrm{~cm}^{-1}$ have been reported for alkyl substituted polythiophene.[3,4] In contrast, designing stable $n$-doped materials proves to be difficult.[5] Due to the low electronegativities of organic polymers, re-

Dedicated to Professor Paul von Ragué Schleyer on the occasion of his $70^{\text {th }}$ birthday duction potentials required for n-doping are highly negative. N-doped systems are subject to high self-discharge and low electrode cycle life.[6] Since reversible n-dopability is necessary for applicability of organic polymers in devices such as batteries, diodes, and supercapacitors, intensive research is under way with the aim of lowering valence band energies. This would simultaneously improve intrinsic conductivity by decreasing the band gap and increasing the charge carrier concentration.[7] Ultimately the goal of designing metallic organic polymers might be achieved by lowering the conduction band energy.

Theoreticians and experimentalists have developed a range of strategies for decreasing band gaps and improving n-dopability. [8-15,5,16,17] One of those is to attach bridging groups with low lying $\pi^{*}$-orbitals to cis-dithiophene. The polymer of 4-dicyanomethylene-4H-cyclopenta[2,1$\mathrm{b}: 3,4-\mathrm{b}$ '] dithiophene 1 has a band gap of $0.8 \mathrm{eV}, 1 \mathrm{eV}$ smaller than that of polythiophene, and is reversibly n-dopable.[18] 
Because of these promising properties, poly-1 was used to construct a Schottky barrier diode.[19] However, the device showed little rectification. A possible explanation was offered in terms of high bulk resistivity in the n-doped state due to excessive undoping.

Recently 1 was combined with 3,4-ethylenedioxythiophene 2, to form a range of copolymers.[20] This approach afforded the lowest band gap organic polymer synthesized to date. Electrochemical investigations indicate that the band gap of a 3,4-ethylenedioxythiophene rich copolymer is below $0.16 \mathrm{eV}$ and probably close to zero. Its intrinsic conductivity is $10^{-3} \mathrm{~S} \mathrm{~cm}^{-1}, 5$ orders of magnitude higher than that of<smiles>N#CC(C#N)=C1c2ccsc2-c2sccc21</smiles>

1<smiles>S=C1c2ccsc2-c2sccc21</smiles>

3<smiles>S=c1c2ccc(=S)c2c2c(=S)ccc12</smiles>
5

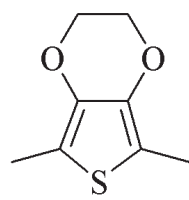

2

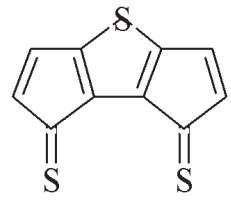

4

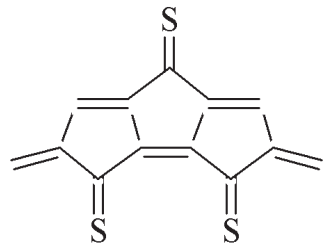

6

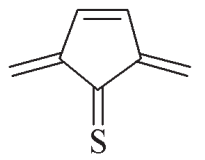

7

Scheme 1 Repeat units for conducting polymers. Polymers of CDM, 1, and of EDOT, 2, are experimentally known low band gap polymers. $\mathbf{3}$ and $\mathbf{4}$ were investigated theoretically and showed promising properties. 5 and $\mathbf{6}$ are focus of the present study. 7 is the smallest building block of 6 and used for comparison poly-1.[21] However, considering that zero band gap polymers are expected to exhibit conductivities larger than $10^{2} \mathrm{~S}$ $\mathrm{cm}^{-1},[8]$ this is still disappointingly low. Analysis by Huang and Pickup[20] revealed that the n-type charge carriers are not extensively delocalized. The reduction potential of $\mathbf{1}$ does not decrease with growing chain length and the reduction potentials of the copolymers do not shift significantly with changing composition. The mobility ratio of $n-$ and p-type carriers in the copolymers is small, 0.002. Thus, the more mobile p-type carriers mainly account for the intrinsic conductivity. The ratio between the mobilities of $n$ - and p-type carriers is larger for poly-1, 0.025, but p-type carriers are still 40 times more mobile than n-type carriers. Therefore, localization of n-type charge carriers appears to be an alternative explanation for the high bulk resistivity of n-doped poly-1 and its failure as a diode material.

Theoretical investigations of poly-1 [14,22] and related systems as well as calculations on the monomeric building blocks[23] support Huang and Pickup's findings. The conduction band widths, which can be very roughly correlated with conductivity,[8] are small for these systems[24] and orbital coefficients at the $\alpha$-carbon atoms of the building blocks are small.[23] Thus, the dicyanomethylene group seems to increase the electron affinity of polythiophene by introducing low lying but localized $\pi^{*}$-orbitals. This means that in the conduction "band", the orbitals do not overlap strongly. Thus poly-1 and its analogs might be a dead end if n-type conductivity is desired.

Hong and Song[14] investigated $\mathrm{C}=\mathrm{S}, \mathrm{C}=\mathrm{O}$, and $\mathrm{C}=\mathrm{CH}_{2}$ bridged cis-dithiophenes with semiempirical solid state calculations. The $\mathrm{C}=\mathrm{S}$ group leads to the smallest band gap. By introducing the above groups alternatively in the outer and in the inner rings (compare $\mathbf{3}$ and $\mathbf{4}$ in Scheme 1), it could be shown that the above groups favor quinoid structures, whereas electron donating atoms like sulfur favor aromatic structures. In the presence of two different bridging groups, the one in the outer ring determines whether the structure is quinoid or aromatic. Thus, poly-3 is aromatic but poly-4 is quinoid. The quinoid polymers have smaller band gaps than the aromatic ones, about $1 \mathrm{eV}$.

We have investigated a range of monomeric derivatives of 1[23] with density functional theory and found that the $\mathrm{C}=\mathrm{S}$ group, in systems such as $\mathbf{3}$, leads to smaller energy gaps than $\mathrm{C}=\mathrm{C}(\mathrm{CN})_{2}$. This might seem surprising at first since the small band gap of poly-1 is usually attributed to the strong electron withdrawing power of the $\mathrm{C}=\mathrm{C}(\mathrm{CN})_{2}$ group. [18] Natural bond orbital analysis revealed that $\mathrm{C}=\mathrm{C}(\mathrm{CN})_{2}$ is not strongly electron withdrawing but owes is large electronegativity to its high electron affinity arising from the presence of a low lying $\pi^{*}$-orbital. This is in line with Mulliken's rather than with Pauling's definition of electronegativity. Thus resonance rather than inductive effects are responsible for the band gap lowering. The similarity between $\mathrm{C}=\mathrm{S}$ and $\mathrm{C}=\mathrm{C}(\mathrm{CN})_{2}$ can be understood by realizing that the $\mathrm{C}=\mathrm{S}$ group also has a low lying $\pi^{*}$-orbital and a high electron affinity. The $\mathrm{C}=\mathrm{S}$ group appears interesting for the design of conducting polymers for several reasons: i) it achieves similar band gap lowering to $\mathrm{C}=\mathrm{C}(\mathrm{CN})_{2}$, ii) it is small enough to be 
incorporated in the all of the rings (5 in Scheme 1) which should result in a quinoid structure with a lower band gap, iii) the $\mathrm{C}=\mathrm{S}$ group should have less capacity for charge trapping than $\mathrm{C}=\mathrm{C}(\mathrm{CN})_{2}$, and finally iv) there is a rich chemistry involving the competition between single and double carbon sulfur bonds so that good mechanical properties due to threedimensional interactions seem possible. Preliminary studies indicated that the polymer of $\mathbf{5}$ might have a very small band gap.[23] Here a more detailed electronic structure analysis of $\mathbf{5}$ and the extrapolation of the oligomer to polymer properties is presented. Extrapolated results for poly- $\mathbf{5}$ are compared to those for polythiophene and poly-1.

\section{Methods}

Monomer through tetramer of $\mathbf{1 , 5}$ and $\mathbf{6}$, and monomer through hexamer of thiophene were optimized in planar geometries using density functional theory (DFT). Becke's three parameter hybrid functional[25] was combined with Perdew and Wang's correlation functional.[26] Stevens Basch Krauss pseudopotentials[27] and split valence plus polarization basis sets were employed.[28] The weight of the Hartree-Fock exchange was increased to $30 \%$ since this functional yields HOMO-LUMO gaps in close agreement with $\lambda_{\max }$ values from UV spectroscopy.[29] IPs and EAs are about $1 \mathrm{eV}$ too low for oligomeric $\pi$-systems but trends are reproduced correctly. This approach is justified from a pragmatic point of view although the meaning of DFT orbital energies is a controversial subject[30-39] that is beyond the scope of the present investigation.

The dimer and all higher oligomers of $\mathbf{5}$ are quinoid and have extremely small energy gaps. A quinoid structure for the polymer was expected according to Hong and Song's[14] work. That the dimer is already quinoid shows that the tendency to switch to a quinoid structure is very strong and that end effects that favor aromatic geometries do not prevent the switch to the quinoid form even for very small oligomers. However, a quinoid structure can not be terminated properly at the ends of the oligomers. Therefore, the wave functions were tested for stability. Not surprisingly, the closed shell wave functions of $\mathbf{5}$ and of its oligomers are singlet unstable. Reoptimization of the singlets with open shell wave functions let to large spin contaminations ( $\mathrm{S}^{2}$ values between 1.1 and 1.8). The energies were only slightly lowered but the energy gaps increased by more than $1 \mathrm{eV}$. With the open shell wave function even the monomer tends to become quinoid. In an attempt to avoid the use of open shell calculations, the oligomers were end-capped with $\mathrm{CH}_{2}$ groups, shown as $\mathbf{6}$ (Scheme 1) and reoptimized. A stability test for monomeric 6 still indicated internal instability, but the spin contamination of the open shell wave function became small $\left(S^{2}=0.1\right)$ and the total energy lowering was only $0.03 \mathrm{kcal} \mathrm{mol}^{-1}$. In an open shell calculation, the energy gap increased by $0.06 \mathrm{eV}$ from $2.81 \mathrm{eV}$ to $2.87 \mathrm{eV}$. This change is negligible compared to the inherent inaccuracy of energy gaps as approximate excitation energies. Therefore the calculations were carried out employing end-capped oligomers and closed shell wave functions. All calculations were performed with Gaussian 94W[40] and 98W,[41] orbital contours were plotted with the g-openmol program.[42]

\section{Results}

\section{Geometries}

Figure 1 shows bond lengths in the inner rings for monomer through tetramer of $\mathbf{6}$. The short bonds connecting the rings and the long bonds where there would be double bonds in thiophene show that the structures are quinoid. As described in the methods section, oligomers of $\mathbf{5}$ are also quinoid despite the influence of end-effects that favor aromatic structures. When starting from an aromatic carbon framework, oligomers of $\mathbf{5}$ optimized smoothly to the quinoid forms, showing that the quinoid structures of oligomers of $\mathbf{6}$ are not caused by the $=\mathrm{CH}_{2}$ groups. In contrast, oligomers of $\mathbf{1}$ are aromatic. Attempts to locate quinoid analogs of dimer and
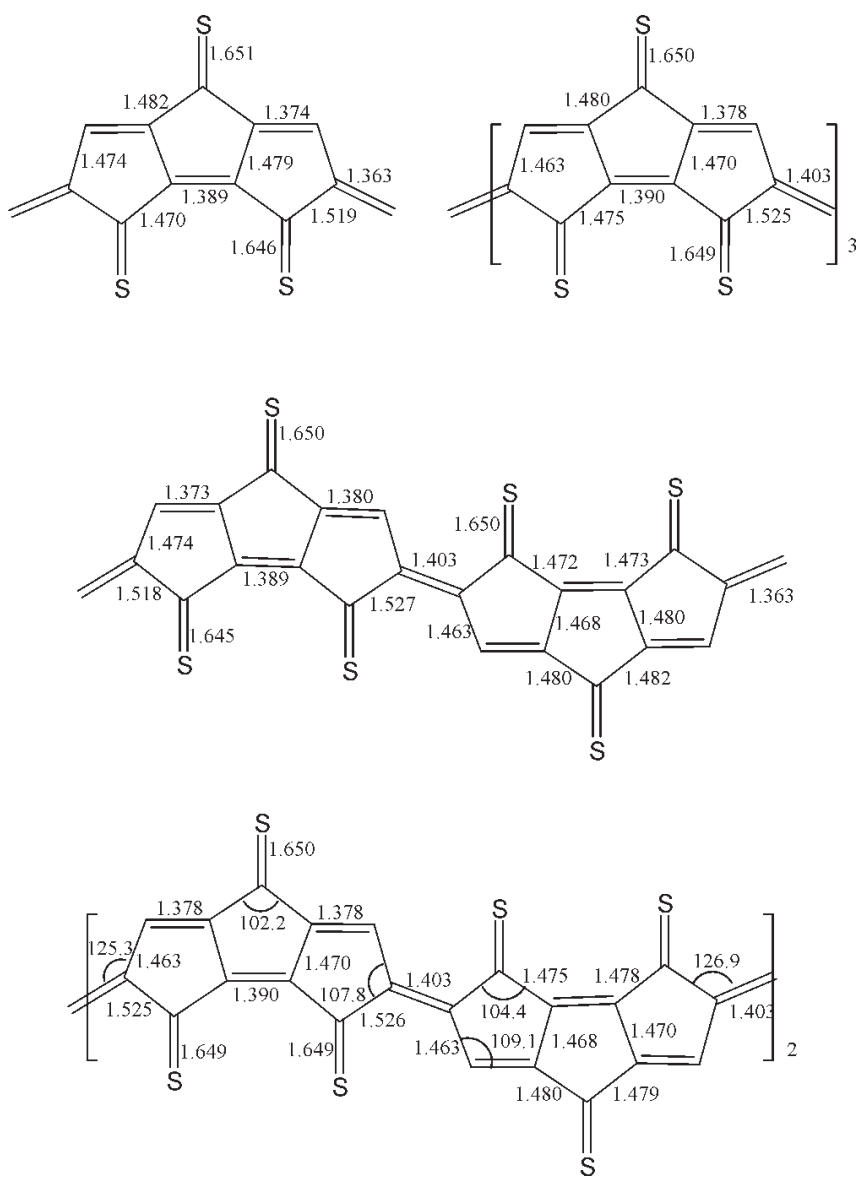

Figure 1 Bond lengths in monomer, dimer, trimer, and tetramer of 6 
Figure 2 Energy levels and development of bands for thiophene 7, and oligomers of 6

Figure 3 Energy levels and development of bands for thiophene, and oligomers of $\mathbf{1}$
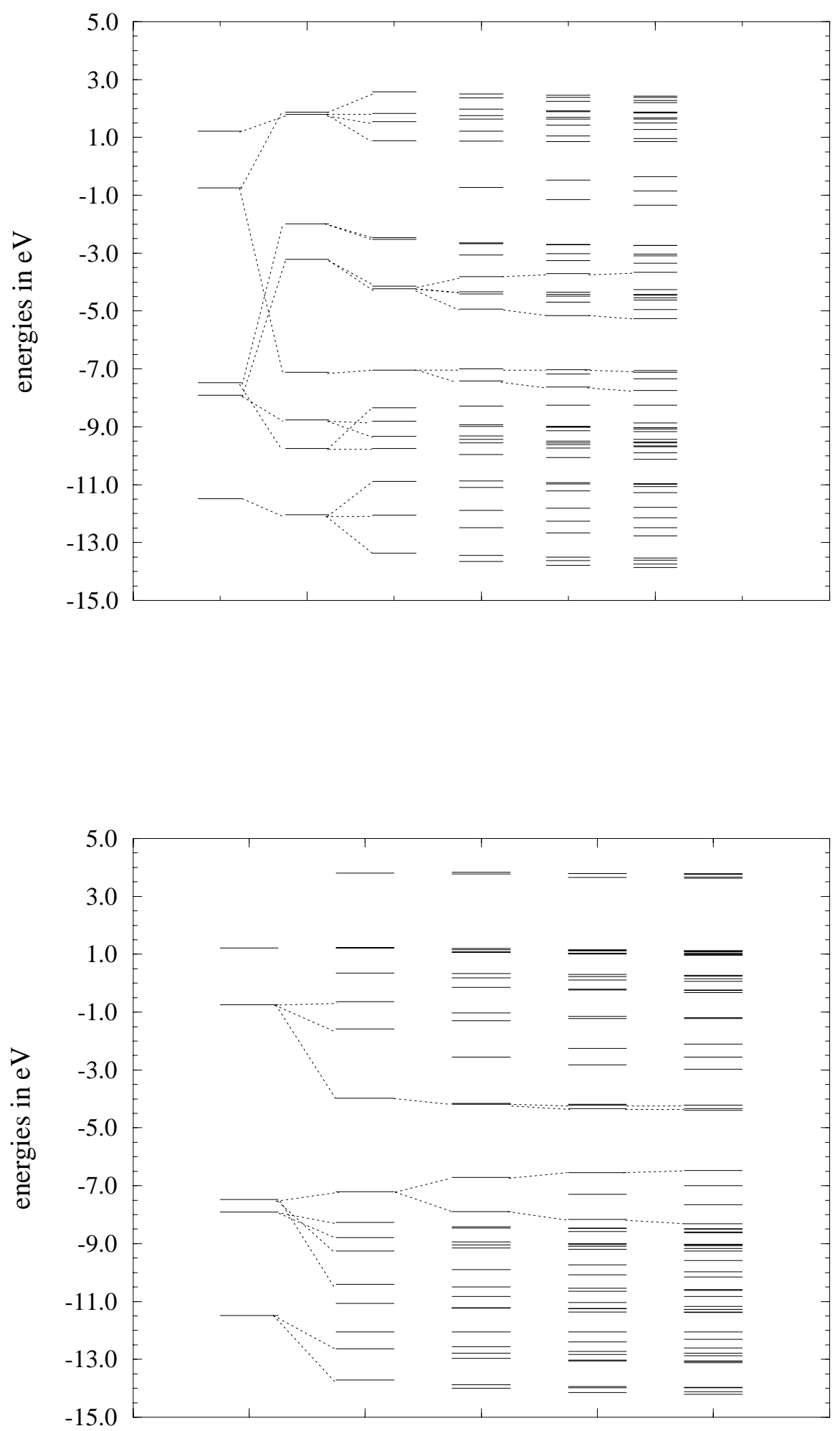
Table 1 Energy levels for oligomers of 6, 1, and thiophene

\begin{tabular}{|c|c|c|c|c|c|}
\hline $\begin{array}{l}\text { \# repeat } \\
\text { units }\end{array}$ & $\begin{array}{l}\text { lower valence } \\
\text { band edge }\end{array}$ & IP & EA & $\begin{array}{l}\text { upper cond. } \\
\text { band edge }\end{array}$ & $\mathbf{E}_{\mathrm{g}}$ \\
\hline \multicolumn{6}{|c|}{6} \\
\hline 1 & \multicolumn{2}{|c|}{-7.04} & \multicolumn{2}{|c|}{-4.22} & 2.82 \\
\hline 2 & -7.41 & -6.99 & -4.93 & -3.81 & 2.06 \\
\hline 3 & -7.62 & -7.02 & -5.15 & -3.71 & 1.87 \\
\hline 4 & -7.75 & -7.05 & -5.26 & -3.66 & 1.79 \\
\hline \multirow[t]{2}{*}{$\infty$} & -8.99 & -7.02 & -5.61 & -3.45 & 1.43 \\
\hline & \multicolumn{2}{|c|}{ Band width: $1.97 \mathrm{eV}$} & \multicolumn{3}{|c|}{ Band width: $2.16 \mathrm{eV}$} \\
\hline
\end{tabular}

\begin{tabular}{lll}
1 & \multicolumn{3}{c}{} \\
2 & -7.89 & -6.72 \\
3 & -8.17 & -6.55 \\
4 & -8.32 & -6.48 \\
$\infty$ & -8.66 & -6.23 \\
\multicolumn{2}{l}{} \\
\hline
\end{tabular}

1

\begin{tabular}{lll}
1 & \multicolumn{3}{c}{-7.48} & -6.59 \\
2 & -7.94 & -6.22 \\
3 & -7.98 & -6.04 \\
4 & -8.07 & -5.93 \\
5 & -8.06 & -5.86 \\
6 & -8.13 & -5.49 \\
$\infty$ & -8.36 & Band width: 2.23 eV \\
& (evaluated for polydithiophene)
\end{tabular}

Thiophene

\begin{tabular}{|c|c|c|}
\hline \multicolumn{2}{|c|}{-3.98} & 3.23 \\
\hline-4.19 & -4.15 & 2.53 \\
\hline-4.34 & -4.19 & 2.21 \\
\hline-4.40 & -4.22 & 2.08 \\
\hline-4.51 & -4.30 & 1.72 \\
\hline
\end{tabular}

Band width: $0.21 \mathrm{eV}$

\begin{tabular}{|c|c|c|}
\hline & & 6.73 \\
\hline-1.78 & -0.10 & 4.81 \\
\hline-2.22 & 0.18 & 4.00 \\
\hline-2.46 & 0.33 & 3.58 \\
\hline-2.61 & 0.42 & 3.32 \\
\hline-2.71 & 0.49 & 3.15 \\
\hline-3.21 & 0.80 & 2.28 \\
\hline
\end{tabular}

Band width: $1.90 \mathrm{eV}$

(evaluated for polydithiophene)

trimer of $\mathbf{1}$ failed likewise. There is thus no indication of the existence of two stable forms of either polymer.

The double bonds connecting the rings of oligomers of 6 are $1.403 \AA$ long, the double bonds within the rings are shorter, $1.378 \AA$. The single bond lengths in the inner rings are 1.468 and $1.470 \AA$. Differences between the inner part of dimer and the trimer are vanishingly small. Thus, bond lengths are essentially converged in the inner rings of the trimer. The maximum bond lengths alternation for poly-6 is predicted to be $0.092 \AA$. For comparison, bond lengths alternation is $0.060 \AA$ in sexithiophene[43] and in quater-1. Thus quinoid poly-6 does not have smaller bond length alternation than aromatic polythiophene and poly-1.

\section{Ionization Potentials (IPs) and Electron Affinities (EAs)}

In Figures 2 and 3 energy levels are plotted for monomer through tetramer of $\mathbf{6}$ and of $\mathbf{1}$, respectively. Figure 4 shows energy levels of thiophene monomer through hexamer. To assign orbitals of the oligomers to bands of the polymers, orbital contours were plotted for the oligomers and visually correlated with those of 7 and of thiophene. These correlations are indicated with dotted lines in Figures 2-4. IPs and
EAs are summarized and extrapolated to polymer values in Table 1. The HOMO levels, and therefore the IPs according to Koopmans' theorem,[44] of oligomers of $\mathbf{6}$ do not decrease with increasing chain length. The IP of poly-6 is predicted to be about $7 \mathrm{eV}, 1.5 \mathrm{eV}$ larger than that of polythiophene and $0.8 \mathrm{eV}$ larger than that of poly- $\mathbf{1}$ at the same level of theory. The extrapolated EA of poly-6 is $5.61 \mathrm{eV}, 2.4 \mathrm{eV}$ larger than that of polythiophene and $1.1 \mathrm{eV}$ larger than that of poly-1 which is a known n-dopable polymer.

\section{Band gaps}

Oligomers of $\mathbf{6}$ have smaller energy gaps than oligomers of $\mathbf{1}$ and of thiophene. Plotting the values against $1 / n$ and extrapolating to infinite chain length (Figure 5) predicts the band gaps of poly-6, poly-1, and polythiophene to be 1.41 $\mathrm{eV}, 1.72 \mathrm{eV}$, and $2.30 \mathrm{eV}$, respectively. Experimental band gaps of poly-1 and of polythiophene are $0.8 \mathrm{eV}[18]$ and 1.8 eV.[45] $\lambda_{\max }$ for ultrathin polythiophene films is $2.30 \mathrm{eV}$,[4] $0.6 \mathrm{eV}$ higher than the band gap value which corresponds to the onset of absorption. Theoretical estimates from band structure calculations correspond to $\lambda_{\max }$ and are therefore in excellent agreement with experiment for polythiophene. As- 
Figure 4 Energy levels and development of bands for oligomers of thiophene

Figure 5 Energy gaps vs 1/ number of repeat units of $\mathbf{6}$, of 1 , and of thiophene (from bottom)
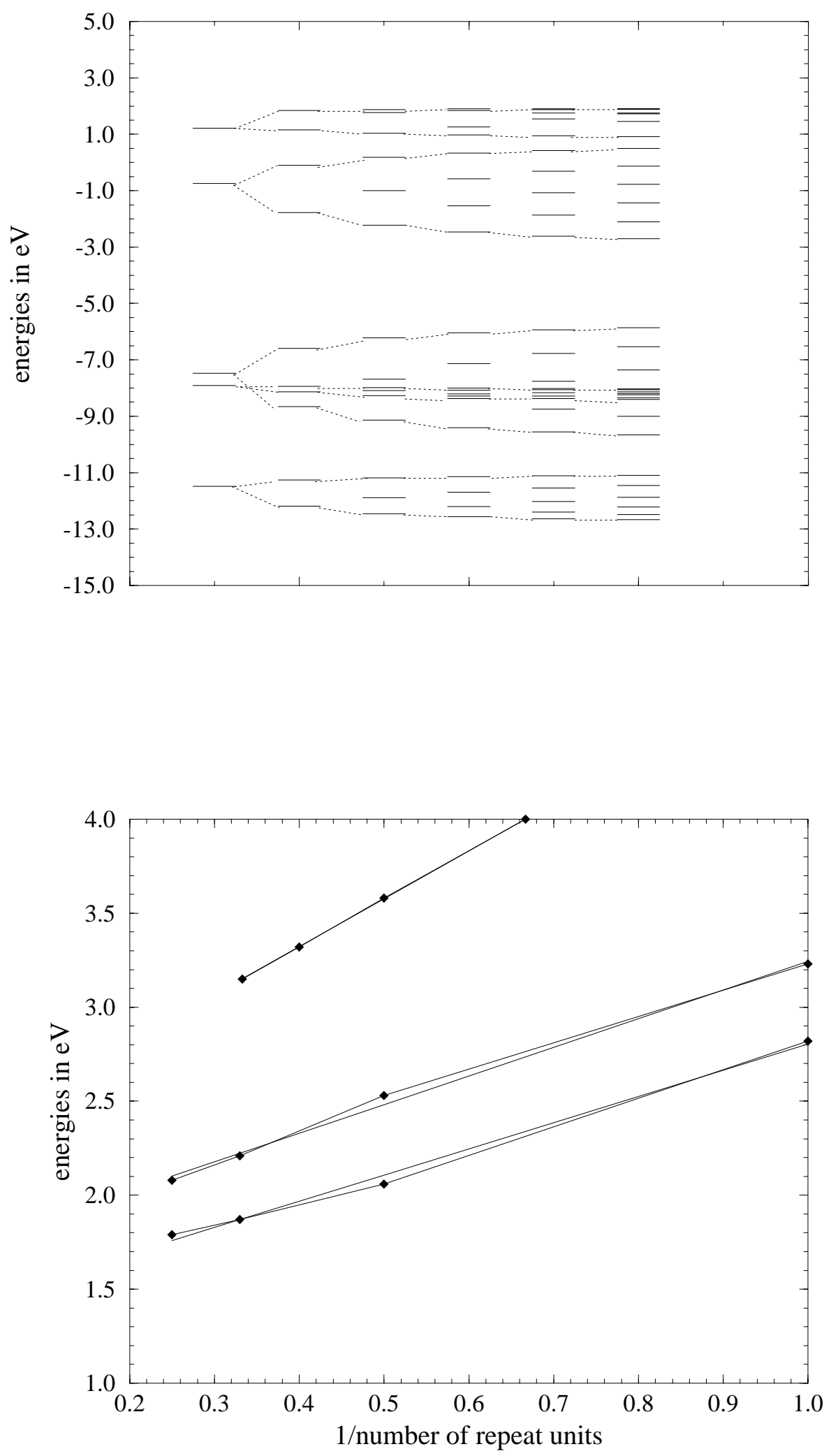


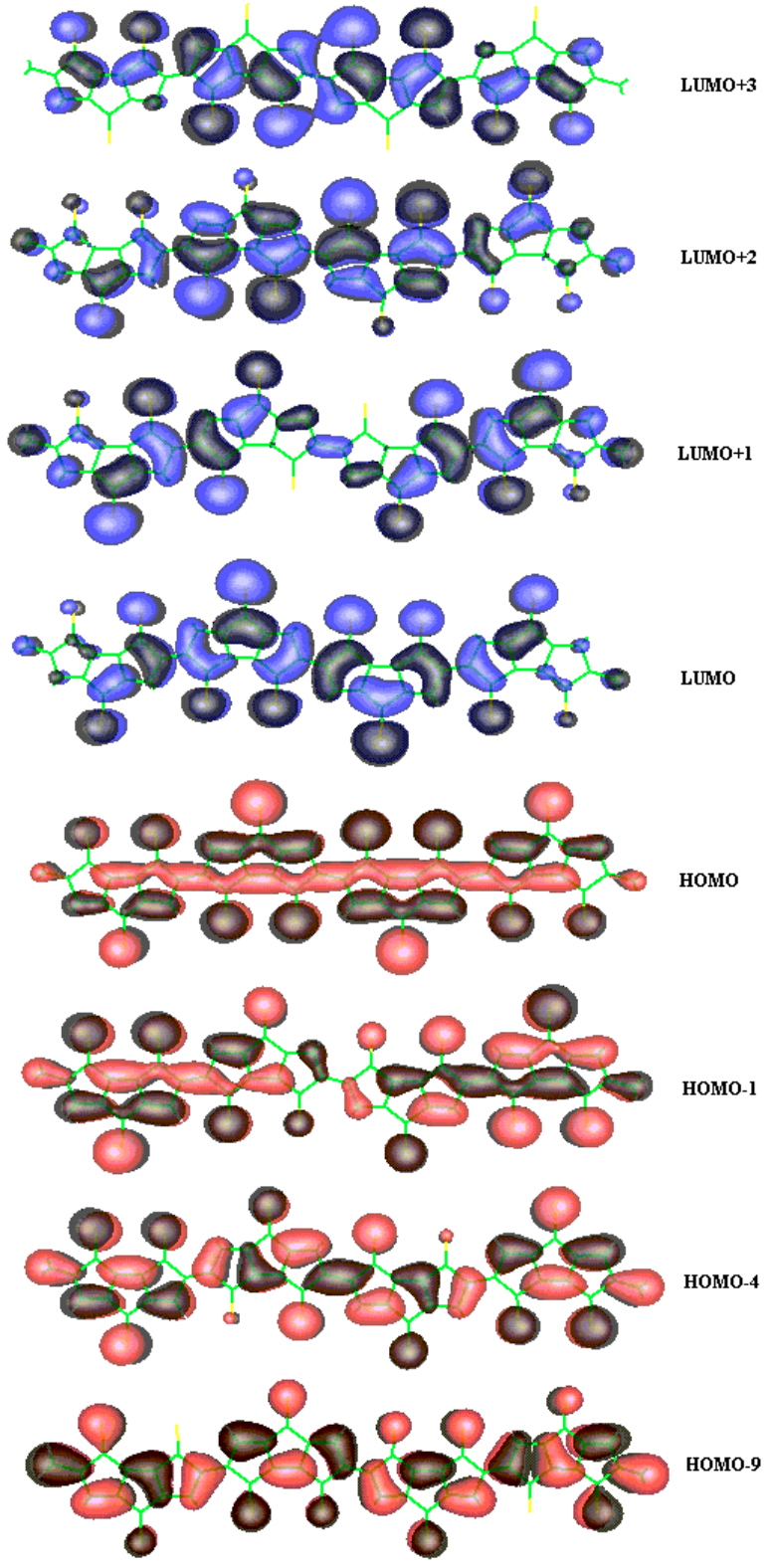

Figure 6 Highest lying occupied (red and black) and lowest lying unoccupied (blue and black) $\pi$-orbitals of quater- 6

suming a similar difference between onset of absorption and $\lambda_{\max }$ for poly-1, our calculations predict a band gap of $1.1 \mathrm{eV}$ for poly-1, $0.3 \mathrm{eV}$ above the experimental value. Therefore, the band gap of poly-6 is estimated to be about $0.5-0.8 \mathrm{eV}$.

\section{Band widths}

Tracing back the bands of quarter-6 shows that its valence band arises from the thiophene LUMO which splits into two levels in quinoid 7, the lower one leading to the HOMO of 6 and giving rise to the valence band of poly-6. The conduc-
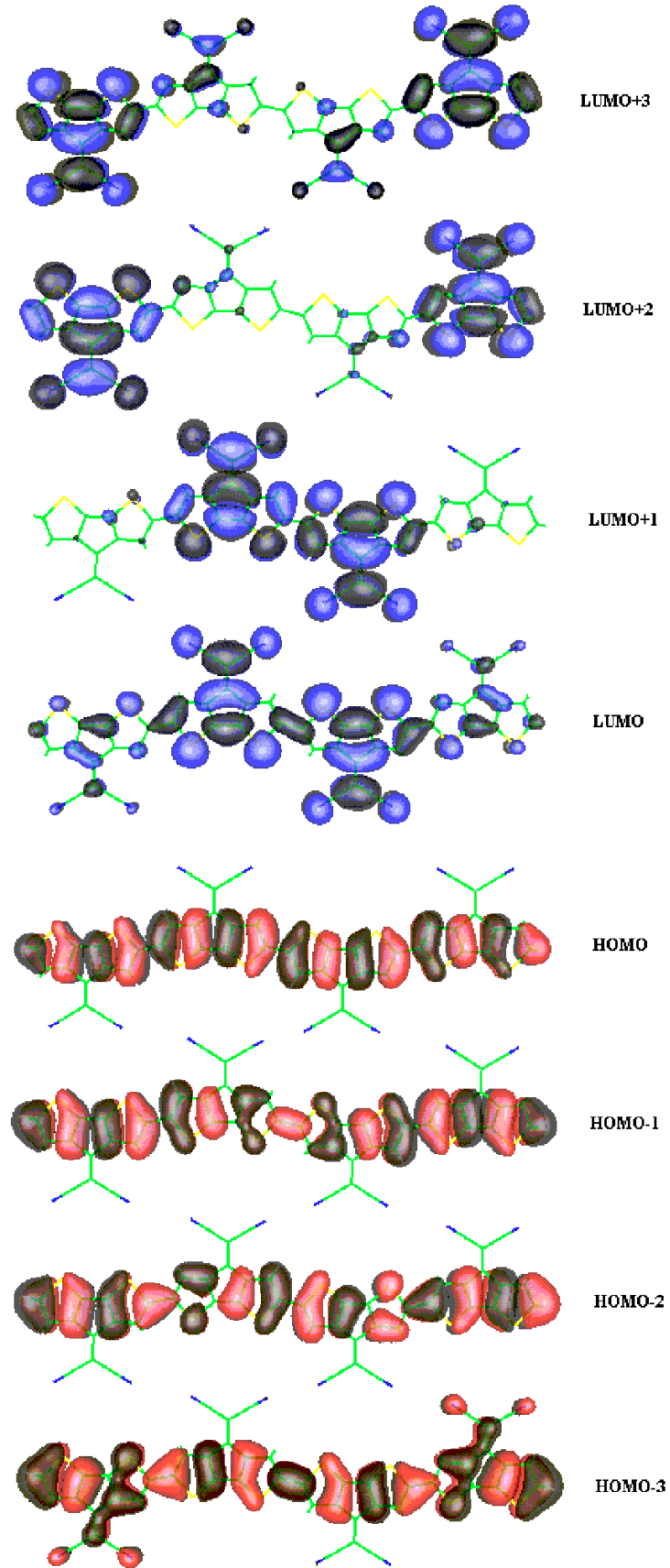

Figure 7 Highest lying occupied (red and black) and lowest lying unoccupied (blue and black) $\pi$-orbitals of quater-1

tion band stems from the HOMO-1 of thiophene, which splits into two levels in 7 . The higher one gives rise to two closely spaced levels in $\mathbf{6}$, one of which forms a wide band, the conduction band, the other one forms a flat band that lies within the conduction band. Thus valence and conduction bands of 6 have different character than those of polythiophene (compare Figures 6 and 8). Both bands are delocalized (compare Figure 6) and have substantial band widths. Extrapolated 


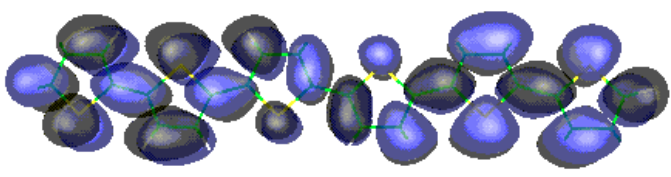

LUMO +2

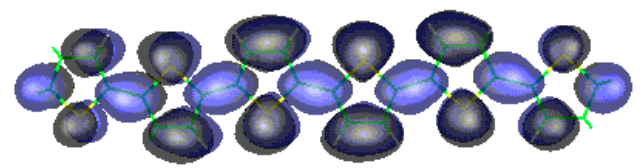

LUMO

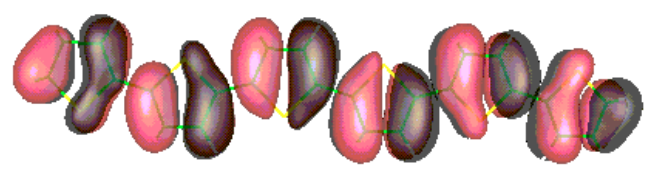

Hомо

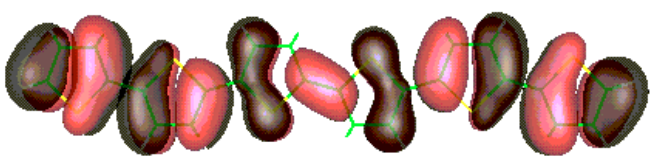

Hомо-1

Figure 8 Highest lying occupied (red and black) and lowest lying unoccupied (blue and black) $\pi$-orbitals of sexithiophene

values are $1.97 \mathrm{eV}$ and $2.16 \mathrm{eV}$, for valence and conduction bands, respectively.

The valence band of poly-1arises from the thiophene HOMO and is practically identical to that of polythiophene, since there are nodes at the positions of the dicyanomethylene groups (compare Figures 7 and 8). Extrapolation leads to a band width of $2.43 \mathrm{eV}$. The conduction band originates in a bonding combination of the thiophene LUMO and the dicyanomethylene group. With increasing chain lengths, the contribution from the dicyanomethylene group leads to localization of the charge density and little overlap between the repeat units (compare the four black and blue colored antibonding orbitals in Figure 7) Therefore the conduction band widths is very small, $0.21 \mathrm{eV}$.

The valence and conduction bands of polythiophene arise from thiophene HOMO and LUMO. Both orbital types lead to significant overlap and wide bands. To compare band widths of poly-6 and poly-1 to those of polythiophene, dithiophene has to be used as the repeat unit to assure that the sizes of the repeat units are comparable. The reason for this is that, in solid state physics terms, doubling of the repeat unit leads to halving of the Brillouin zone. The smaller Brillouin zone contains twice as many bands of smaller band widths. With dithiophene as a repeat unit, valence and conduction band widths of polythiophene are $2.23 \mathrm{eV}$ and $1.90 \mathrm{eV}$. Thus polythiophene and poly-6 have comparable valence and conduction band widths. The valence band of poly-1 is also wide, only the conduction band of poly-1 stands out by being extremely narrow.

\section{Discussion}

The basic idea behind conducting polymers is that infinite chains of conjugated $\pi$-systems act as molecular wires, allowing charge transport through the delocalized $\pi$-system. In such a wire, conductivity is related to charge carrier concentration and mobility of charge carriers. [8] Charge carriers are both electrons and holes and the resulting conductivity is the sum of their contributions. [46] The charge carrier concentration at ambient temperature or in the presence of light increases as the band gap decreases. Alternatively, doping can create charge carriers. The charge carrier mobility along the chain increases with increasing overlap between repeat units, which is reflected in the band width. In real systems, band widths correlate only very roughly with mobilities of charge carriers since chains are not infinite and charge carriers have to hop.[8] That real polymers are far from molecular wires can be seen by the fact that properties of polythiophene can be very well modeled with sexithiophene oligomers, which in the crystalline state conduct perpendicular to the chain direction rather than along the chains.[47] On the other hand, the dramatic increase in conductivity of polyacetylene after stretch aligning[48] and the improved conductivity of more ordered ultrathin polythiophene films $[3,4]$ shows that the molecular wire picture is valid if the bulk properties of the material can be optimized. Thus, small band gaps and large band widths are necessary though not sufficient conditions for good conductivity.

Poly-1 is one of the lowest band gap conducting polymers synthesized to this date, copolymers of $\mathbf{1}$ and 2[20] might be the first zero band gap materials produced since the discovery of polysulfurnitride. The low band gaps of these systems are due to the presence of the $\pi$-system of the dicyanomethylene group, which provides for a low lying LUMO. However, although poly-1 is repeatedly n-dopable, the conductivity in the n-doped state is low.[19] Poly-1 has not been investigated as thoroughly as polythiophene. The crystal structure has not been determined for oligomers. Conjugation lengths and film thickness have not been optimized. Therefore, disorder could be responsible for the low intrinsic conductivity and the low conductivity of the n-doped forms. Low chemical stability of the n-doped form[19] is another possibility. One factor, however, seems to have been overlooked. The conduction band of poly-1 is very flat. This indicates little overlap between the repeat units. Figure 7, which displays orbital contours of conduction band orbitals, clearly confirms the relation between low overlap and small band width. In other words the conduction band is not a band but consists of individual localized orbitals. Dicyanomethylene groups might act as traps for the electrons and thus prevent conductivity contributions from n-type carriers. Copolymers of $\mathbf{1}$ and $\mathbf{2}$ might have zero band gaps but still their intrinsic conductivities are small. The n-type carrier mobility is 500 times smaller than that of the p-type carriers.[20] Thus poly-1 and copolymers containing $\mathbf{1}$, might be intrinsically poor conductors in the $n$-doped state even if the n-doped forms were chemically stable and if the chains were infinite and perfectly aligned. This seems to 
be in agreement with findings of Huang and Pickup that the conduction band energy did not change much with composition of the copolymers.[20] Disorder or short conjugation lengths should limit mobilities of both, p- and n-type carriers and can not be responsible for these huge differences. Excessive dedoping can explain the low conductivity of n-doped poly-1, it can however, not account for the low intrinsic conductivity. Thus, localization of n-type charge carriers remains as possible reason for the low conductivity of neutral and ndoped poly-1.

Neither poly-6 nor its building blocks have been synthesized. Therefore it can only be guessed whether it might be a stable substance. The smallest unit of $\mathbf{6}$ is thiocyclopentadieneone, the sulfur analogon of cyclopentadieneone. Cyclopentadieneone is known but very reactive. The reason for this is its antiaromatic character. Due to the polarity of the $\mathrm{C}=\mathrm{O}$ bond, it is essentially an antiaromatic $4 \pi$-electron system. Since sulfur is less electronegative, the polarity of the $\mathrm{C}=\mathrm{S}$ bond is opposite to that of $\mathrm{C}=\mathrm{O}$ and thiocyclopentadieneone is closer to an aromatic system that could be more stable. The IPs of $\mathbf{6}$ and its oligomers are quite high, thus spontaneous electron loss upon standing in air as in the case of polypyrrole is not expected. The high IP of poly-6 might, in fact make p-doping difficult. The neutral form of poly-6 might thus be a stable compound.

The best estimate for the band gap of poly- 6 is about 0.5 $0.8 \mathrm{eV}$, similar to that of $\mathbf{1}$. The charge carrier concentration at room temperature or after illumination should therefore be similar in the two polymers. However, the EA of poly- 6 is predicted to be about $1.1 \mathrm{eV}$ higher than that of poly-1. Thus if low chemical stability of the n-doped form of poly-1 indeed contributes to its low n-type conductivity, poly-6 could show improved performance. The conduction band of poly- 6 is not as flat as that of poly-1. This should make n-type carriers more mobile along the chains. The more delocalized nature of the orbitals in the conduction band can be seen in Figure 6. Thus if localization of n-type charge carriers in poly-1 is the reason for the low n-type conductivity of poly1, poly-6 would again be superior. Therefore, poly-6 appears to be a good candidate for an n-dopable conducting polymer with significant intrinsic conductivity along the chain. Gas phase calculations cannot make predictions about limitations due to short conjugation length, disorder and large exciton binding energy. We have not tried to examine hopping effects between chains. The presented calculations can therefore only suggest that poly-6 fulfils the some necessary conditions for good conductivity, they cannot guarantee it.

\section{Conclusions}

Electronic structure analysis of poly- $\mathbf{6}$ and poly-1 predicts that there are some interesting differences between the two polymers. In agreement with experiment and semiempirical solid state calculations our results show that poly-1 has a low band gap and a large electron affinity but a very flat conduction band. The conduction band of poly-6 is predicted to lie about $1.1 \mathrm{eV}$ lower and to be much wider. Thus poly-6 could have higher stability and higher conductivity in the n-doped state. These consideration apply to ideal polymers of infinite chain length and perfect order. Factors that limit conductivity, such as short conjugation lengths, disorder, unsuitable packing in the bulk, and high exciton binding energies are beyond the scope of the present investigation.

Acknowledgment The author wishes to thank Bilkent University for providing computational resources.

\section{References}

1. Handbook of Conducting Polymers; 2nd ed.; Skotheim, T. A.; Elsenbaumer, R. L.; Reynolds, J. R., Eds.; Marcel Dekker, Inc.: New York, 1997.

2. Walatka, V. V.; Labes, M. M.; Perlstein, J. H. Phys. Rev. Lett. 1973, 31, 1139.

3. Roncali, J.; Yassar, Y.; Garnier, F. J. Chem. Soc., Chem. Commun. 1988, 581.

4. Yassar, A.; Roncali, J.; Garnier, F. Macromolecules 1989, 22, 804.

5. Roncali, J. Chem. Rev. 1997, 97, 173.

6. Arbizzani, C.; Catellani, M.; Matragostino, M.; Mingazzini, C. Electrochim. Acta 1995, 40, 1871.

7. Beyer, R.; Kalje, M.; Kingscote-Burton, G.; Murphy, P. J.; Pereira, V. M. S. C.; Taylor, D. M.; Williams, G. O. Synth. Met. 1998, 92, 25.

8. André, J.-A.; Delhalle, J.; Brédas, J.-L. Quantum Chemistry Aided Design of Organic Polymers. An Introduction to the Quantum Chemistry of Polymers and its Applications; World Scientific: London, 1991.

9. Havinga, E. E.; ten Hoeve, W.; Wynberg, H. Synth. Met. 1993, 55-57, 299.

10. Hong, S. Y.; Kwon, S. J.; Kim, S. C.; Marynick, D. S. Synth. Met. 1995, 69, 701.

11. Kertesz, M. Synth. Met. 1995, 69, 641.

12. Kertesz, M.; Hughbanks, T. R. Synth. Met. 1995, 69, 699.

13. Bakhshi, A. K.; Yamaguchi, Y.; Ago, H.; Yamabe, T. Synth. Met. 1996, 79, 115.

14. Hong, S. Y.; Song, J. M. J. Chem. Phys. 1997, 107, 10607.

15. Hong, S. Y.; Song, J. M. Synth. Met. 1997, 85, 1113.

16. Bakhshi, A. K.; Rattan, P. J. Mol. Struct. (THEOCHEM) 1998, 430, 269.

17. Kertesz, M.; Choi, C. H.; Sun, G. Polym. Prepr. (Am. Chem. Soc., Div. Polym. Chem.) 1998, 39,76.

18. Ferraris, J. P.; Lambert, T. L. J. Chem. Soc., Chem. Commun. 1991, 1268.

19. Gunatunga, S. R.; Jones, G. W.; Kalaji, M.; Murphy, P. J.; Taylor, D. M.; Williams, G. O. Synth. Met. 1997, 84, 973.

20. Huang, H.; Pickup, P. G. Chem. Mater. 1998, 8, 2212.

21. Huang, H.; Pickup, P. G. Acta Polym. 1997, 48, 455.

22. Bakhshi, A. L.; Rattan, P. J. Chem. Soc., Faraday Trans. 1998, 94, 2823.

23. Salzner, U.; Lagowski, J. B.; Poirier, R. A.; Pickup, P. G. J. Org. Chem. 1999, 64, 7419. 
24. Hong, S. Y.; Kwon, S. J.; Kim, S. C. J. Chem. Phys. 1995 $103,1871$.

25. Becke, A. D. Phys. Rev. A 1988, 38, 3098.

26. Perdew, J. P. Phys. Rev. B 1986, 33, 8822.

27. Stevens, W.; Basch, H.; Krauss, J. J. Chem. Phys. 1984, $81,6026$.

28. Frisch, M. J.; Frisch, Æ.; Foresman, J. B. Gaussian 94 User's Reference; Gaussian, Inc.: Pittsburgh, 1994-1995.

29. Salzner, U.; Lagowski, J. B.; Pickup, P. G.; Poirier, R. A. J. Phys. Chem. 1998, 102, 2572.

30. Perdew, J. P.; Levy, M. Phys. Rev. Lett. 1983, 51, 1884.

31. Williams, A. R.; von Barth, U. In Theory of the Inhomogeneous Electron Gas; Lundqvist, S., March, N. H., Eds.; Plenum Press: London, 1983.

32. Godby, R. W.; Schlüter, M.; Sham, L. J. Phys. Rev. B 1988, 37, 10159.

33. Fritsche, L. Physica B 1991, 172, 7.

34. Levy, M. Phys. Rev. A 1995, 52, 50.

35. Galbraith, J. M.; Schaefer, H. F. I. J. Chem. Phys. 1996, $105,862$.

36. Baerends, E. J.; Gritsenko, O. V. J. Phys. Chem. A 1997, 101, 5383.

37. Chan, G. E.-L.; Tozer, D. J.; Handy, N. C. J. Chem. Phys. 1997, 107, 1536.

38. Rösch, N.; Trickey, S. B. J. Chem. Phys. 1997, 106, 8940.

39. Savin, A.; Umrigar, C. J.; Gonze, X. Chem. Phys. Lett. 1998, 288, 391.

40. Gaussian 94, Revision B.3; Frisch, M. J.; Trucks, G. W.; Schlegel, H. B.; Gill, P. M. W.; Johnson, B. G.; Robb, M. A.; Cheeseman, J. R.; Keith, T.; Petersson, G. A.; Montgomery, J. A.; Raghavachari, K.; Al-Laham, M. A.; Zakrzewski, V. G.; Ortiz, J. V.; Foresman, J. B.; Peng, C. Y.; Ayala, P. Y.; Chen, W.; Wong, M. W.; Andres, J. L.;
Replogle, E. S.; Gomperts, R.; Martin, R. L.; Fox, D. J.; Binkley, J. S.; Defrees, D. J.; Baker, J.; Stewart, J. P.; Head-Gordon, M.; Gonzalez, C.; Pople, J. A.; Gaussian, Inc.: Pittsburgh PA, 1995.

41. Gaussian 98, Revision A.3; Frisch, M. J.; Trucks, G. W.; Schlegel, H. B.; Scuseria, G. E.; Robb, M. A.; Cheeseman, J. R.; Zakrzewski, V. G.; Montgomery, J., J. A.; Stratmann, R. E.; Burant, J. C.; Dapprich, S.; J. M. Millam; Daniels, A. D.; Kudin, K. N.; Strain, M. C.; Farkas, O.; Tomasi, J.; Barone, V.; Cossi, M.; Cammi, R.; Mennucci, B.; Pomelli, C.; Adamo, C.; Clifford, S.; Ochterski, J.; Petersson, G. A.; Ayala, P. Y.; Cui, Q.; Morokuma, K.; Malick, D. K.; Rabuck, A. D.; K. Raghavachari; Foresman, J. B.; Cioslowski, J.; Ortiz, J. V.; Stefanov, B. B.; Liu, G.; Liashenko, A.; Piskorz, P.; Komaromi, I.; Gomperts, R.; Martin, R. L.; Fox, D. J.; Keith, T.; Al-Laham, M. A.; Peng, C. Y.; Nanayakkara, A.; Gonzalez, C.; Challacombe, M.; Gill, P. M. W.; Johnson, B.; Chen, W.; Wong, M. W.; Andres, J. L.; Gonzalez, C.; Head-Gordon, M.; Replogle, E. S.; Pople, J. A.; Gaussian, Inc.: Pittsburgh PA, 1998.

42. Laaksonen, L. Finnish gOpenmol Efford; http:// laaksonen.csc.fi/gopenmol/, 1999.

43. Salzner, U.; Lagowski, J. B.; Poirier, R. A.; Pickup, P. G. Synth. Met. 1998, 96, 177.

44. Koopmans, T. A. Physica 1934, 1, 104.

45. Chen, T.-A.; Rieke, R. D. Synth. Met. 1993, 60, 175.

46. Kittel, C. Introduction to Solid State Physics; Wiley \& Sons: New York, 1976

47. Wu, M. W.; Conwell, E. M. Chem. Phys. Lett. 1997, 266, 363.

48. Naarmann, C. H.; Theophilou, N. Synth. Met. 1987, 22, 1. 